\title{
An on-line path planning of assembly robots based on FOVS
}

\section{Mingzhou Liu}

Hefei University of Technology

Xin Xu

Hefei University of Technology

Xiaoqiao Wang ( $\nabla$ wxq20061087@163.com )

Hefei University of Technology https://orcid.org/0000-0003-0807-9203

\section{Maogen Ge}

Hefei University of Technology

Lin Ling

Hefei University of Technology

Conghu Liu

Suzhou University

\section{Original Article}

Keywords: Field of view space, Mechanical Product Assembly, Path Planning, Ant Colony Algorithms, Robots

Posted Date: April 8th, 2021

DOI: https://doi.org/10.21203/rs.3.rs-349713/v1

License: (c) (i) This work is licensed under a Creative Commons Attribution 4.0 International License.

Read Full License 


\title{
An on-line path planning of assembly robots based on FOVS
}

\author{
Mingzhou Liu ${ }^{1}$, Xin Xu ${ }^{1}$, Xiaoqiao Wang ${ }^{1}$, Maogen Ge ${ }^{1}$, Lin Ling ${ }^{1}$, Conghu Liu ${ }^{2}$
}

\begin{abstract}
:
To improve the accuracy and efficiency of path planning for the mechanical assembly process of products, an on-line path planning method for mechanical assembly process robots based on visual field space is proposed in this paper. Firstly, to predict and describe the assembly process, the concept of field-of-view space (FOVS) is proposed. Secondly, image processing is carried out by knowledge base to judge the assembly type and current assembly state, and the initial assembly path is given. Then, the assembly process is integrated and solved, and the location estimation of obstacles are given according to the FOVS. Finally, the ant colony algorithm is improved to get the final assembly optimization path. Comparing the algorithm with the ACS algorithm in the aspect of path planning. The length of path planning is reduced by $2 \%$, and the algorithm time is reduced by $0.5 \mathrm{~s}$, the accuracy and efficiency have been effectively improved. the result shows that the algorithm is effective.
\end{abstract}

Keywords: Field of view space, Mechanical Product Assembly, Path Planning, Ant Colony Algorithms, Robots

\section{Introduction}

Recently, with the proposal of the continuous development of industrial robotics technology, unmanned operation is becoming the inevitable trend of the future development of the manufacturing industry [1-3]. With the improvement of the manufacturing level, robots must adapt to the new conditions in the unstructured environment, and they must also use their wisdom adaptively and effectively. In the modern manufacturing industry, the workload of the assembly process accounts for $20 \%$ to $70 \%$ of the whole manufacturing process, and the cost of assembly process accounts for $40 \%$ or more of the production cost. Flexible automatic assembly has always been a major difficulty in manufacturing automation [4].

At present, the assembly process automation of small variety and large batch is widely used, but the automation of small batch and multi variety assembly process is rarely used. There is environmental uncertainty in the assembly process of small batch and multi variety. This uncertainty includes two points. On the one hand, to meet the requirements or avoid obstacles, assembly path planning is an important part of

\footnotetext{
$\triangle$ Xiaoqiao Wang

wxq20061087@163.com
}

1. School of Mechanical Engineering, Hefei university of technology, Hefei, 230009, china;

2. a. Sino-US Global Logistics Institute, Shanghai Jiao Tong University, Shanghai, 200030, China; b. School of Mechanical and Electronic Engineering, Suzhou University, Suzhou, 234000, China. 
assembly operations, and the solution of assembly path planning can also be used to verify the rationality of product design and assembly sequence planning. On the other hand, as the working object will change its position because of deformation or offset, it is very difficult for the executing agency to complete the processing of the target object only through teaching. This requires that the executing agencies can perceive and adjust the execution path when the environment changes.

In view of the above two difficulties, this paper obtains the environmental information and work piece information in real time by constructing the field of view space, and plans the initial assembly path, and then optimizes the path by optimizing ant colony algorithm. Aiming at the two problems mentioned in the introduction, the literature review will be carried out according to the two aspects of visual image processing and assembly path optimization.

At present, many machine vision and robot control systems are widely used [5-6]. At the same time, human-computer interaction [7-8], tactile [9], and predicting ergonomic risk [10] have made some progress in robot control. Paper [11-13] are the mature and in-depth application in machine vision. Yao Guo [14] sensed the patient's gait in the home through machine vision, accurately judged the abnormal gait of the person, and let the assistant robot play a very important role in improving the quality of life of the patient at home. Noriaki Hirose [15] proposed a depth vision model predictive control strategy learning method, which can avoid collision with invisible objects in the navigation path while performing visual navigation. He Kaiming [16-20] and Piotr Dollar[21-23] have done a lot of research on visual perception and image processing. Given the bottleneck of region recommendation computation, he proposed a region recommendation network (RPN). It also predicts the object boundary and score of each location. RPN produces high-quality region construction through end-to-end training. In the ILSVRC and COCO 2015 competition, faster R-CNN and RPN are the basis for winning the first place in many competitions. Wei Liu [24] proposed a new method of haze removal before image restoration based on sky segmentation and dark channel to solve the prior defects of the dark channel. This method can effectively restore the Inland Waterway image. Evan Shelhamer [25] defined the full convolution network for semantic segmentation, transformed the existing classification network (Alexnet, VGG network, and google net) into the full convolution network, and transferred the learned representation to the segmentation task through fine-tuning. Then, a skip architecture is defined, which combines the semantic information from deep and rough layers with the appearance information from shallow and fine layers to produce accurate and detailed segmentation. Multi-manipulator [26], mobile robot [27] and UAV [28] related visual 
perception and image processing are also gradually in-depth; in addition to camera recognition, there are also ultraviolet or near-infrared spectroscopy system detection used in visual perception [29]. To sum up, the application mode of machine vision in the field of application is relatively single, lacking of deconstruction of space. The fusion of vision and surrounding multiple information is worth studying

In the field of human-computer interaction and robot assembly, Wang [30] proposes the assembly tasks related to space robots, Shi [31] uses flexible grippers for micro-assembly tasks, $\mathrm{Xu}$ [32] has a good effect on dual-arm robots, human-computer assistance, and isolation. Anouar Benamor [33] proposed a new multi-objective design method for optimal control of robots. The robot system was described as a linear time-varying model. The weight was optimized by a genetic algorithm, which not only reduced the tracking error but also improved the tracking response under the condition of reducing the oscillation. Yue Wang [34] programmed assembly tasks for industrial robots by decomposing human demonstrations into a series of assembly skills and compiling them into robot executable files. Wafa Boukadida [35] mainly studies robust optimal sliding mode control law for uncertain discrete-time robotic systems with high nonlinearity, unmodeled dynamics, and uncertainty. Seyed Mohammad Ahmadi [36] proposed a robust task space control method. The adaptive Taylor series uncertainty estimator was used to estimate the motor manipulator. At the same time, the upper bound of approximation error was estimated to form a robust term. The asymptotic convergence of tracking error and its time derivative was proved based on stability analysis. To improve the sorting accuracy and efficiency of the sorting system of large inertia robots, a new trajectory planning method based on S-shaped acceleration and deceleration algorithm is proposed. It can be seen that vision based multi-objective path planning is still lack of research, and assembly path optimization in dynamic environment is needed.

The rest of the paper is organized as follows. In section 2, we give the overview of methodology and gives specific methods and elaborate on the concept of FOVS and optimization of path planning. Section 3 validates by an example of assembling the lower body of a solenoid valve. The conclusion of the paper is discussed in section 4 .

\section{2. methodology}

\subsection{Overview of methodology}

Visual-based path planning is an effective path planning method so far. It has been widely used in the industrial field because of its many advantages [37]. The main 
advantages are low cost, easy installation, capturing real scene maps and collision detection before actual contact, etc. Therefore, vision-based machine monitoring and guidance is one of the important ways to be applied in all walks of life [38].

In Fig. 1, the environment space includes information of robots, obstacles, machine vision and so on. The target path needs to be planned in a limited space. In order to describe the path planning in the limited space, the Field of View Space (FOVS) is proposed. The FOVS consists of three aspects: the reachable space of robots, the position space of products and parts, and the field of view. Firstly, the product model knowledge base is established by product modeling, and the complete product parameters are given. Secondly, the initial image information is obtained by taking pictures and comparing images, after visual processing algorithm library, the product shape and position direction are identified by edge searching and model library comparison. Then, according to the machine, the product shape and position direction are identified. The motion characteristics and posture of the robot are used to get the actuator status information. After visual information processing, product-related parameters processing, and robot status information processing, multi-source information fusion is carried out, and the real-time path planning model is given, and the initial path is obtained. Then the initial path is validated and optimized according to the relevant algorithm to complete the assembly task in an uncertain environment.

One of the most important directions in the research of robot cooperative environment using vision-guided active collision avoidance system is to improve flexibility and productivity. Therefore, this paper proposes a path planning method for assembly process robot based on FOVS. The space state is judged in real-time by-product process parameters, accurate field-of-view image information, and robot state parameters. The robot is guided to assemble by image information, which reduces the requirement of fixture positioning accuracy and improves assembly efficiency. 

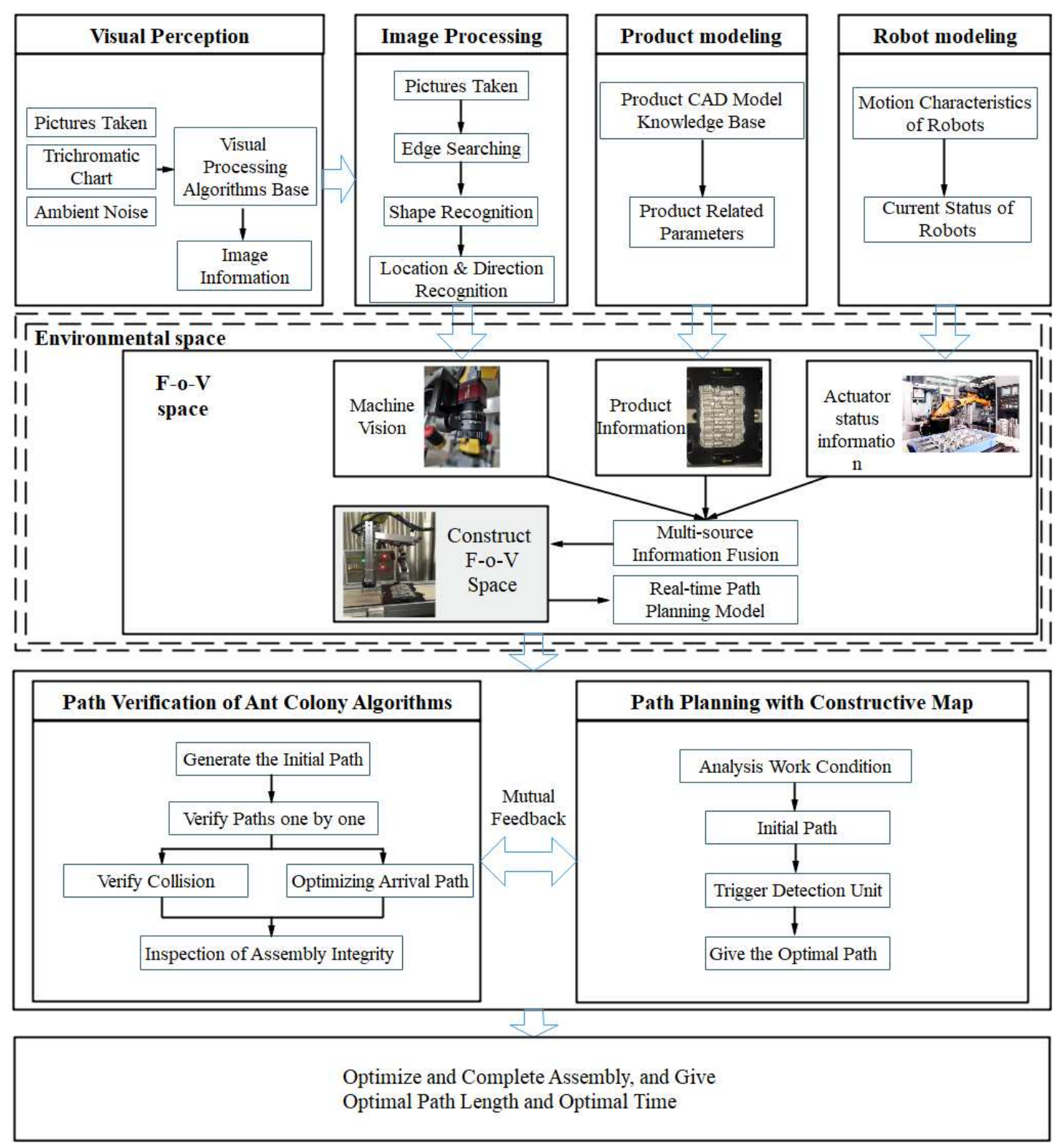

Fig 1 general framework

\subsection{FOVS}

Given how to collect and process different assembly processes in different environments, depending on a single visual field information can not reflect the real situation of the field of view, and considering the assembly process parameters and robot status information, a complete position status information can be obtained. According to the relationship between this information, this paper proposes the concept of field of view space.

As shown in Figure 2, the robot path planning is a dynamic process. This process consists of the Field-of-view expression, Path planning, Application path, Actuator action, System perception module. It is a closed-loop control system. The relationship among product process parameters, robot state information and visual field information 
constitutes the Field-of-view expression. 1, Process parameters of products gives Initial state information and initial path planning. 2, Process parameters of actuator gives Actuator status information and Fixture, Positioning state. 3, Initial visual information and System perception module determine the Update visual information and Obstacle examination and location.

The FOVS gives the spatial model, the type of work piece, the number of target points, the location of obstacles and other information. Firstly, the FOVS can solve the problem of spatial expression of field of view for obstacles or environmental changes, and secondly, it can solve the corresponding relationship among the field of view, actuator and assembly space.

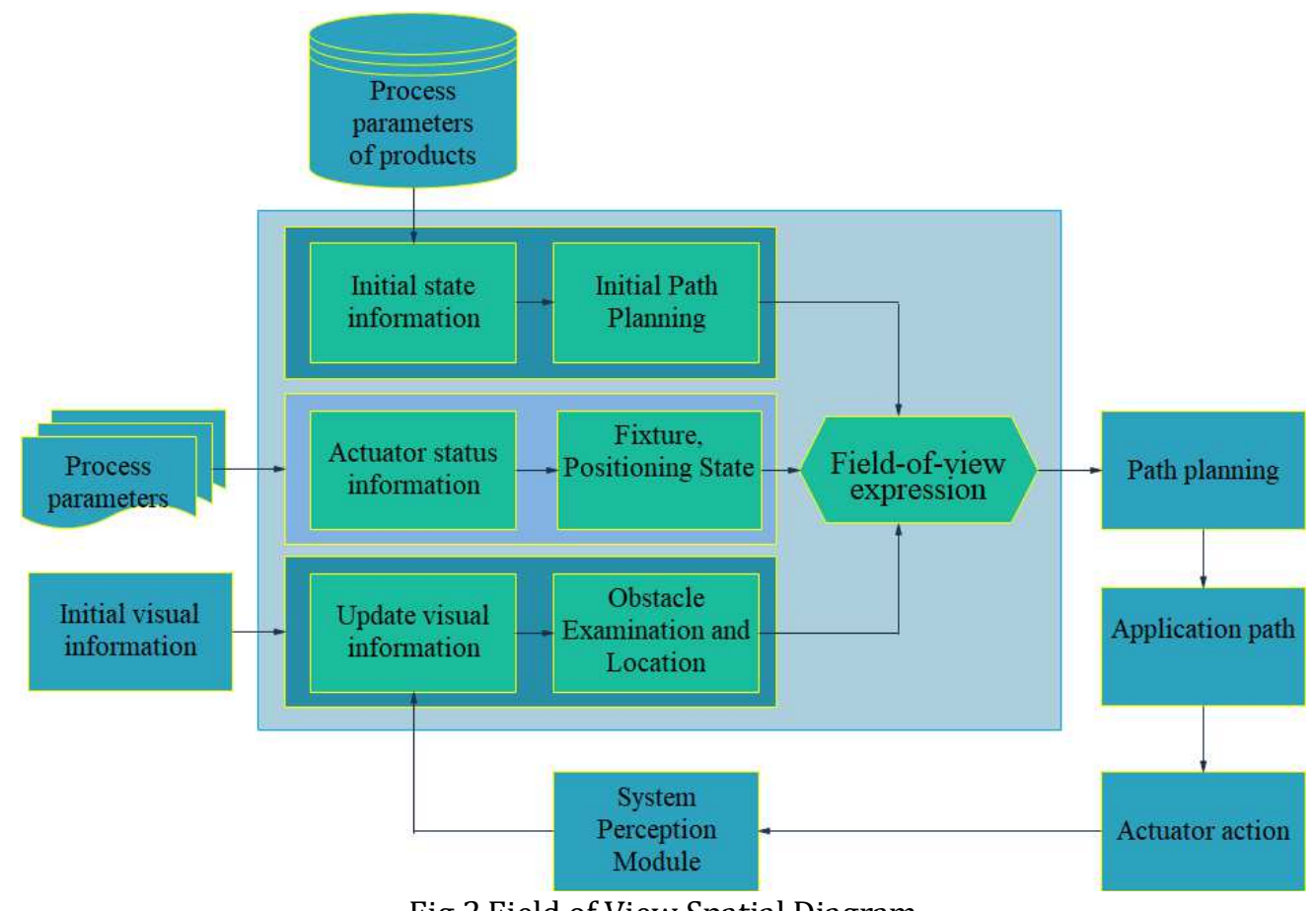

Fig 2 Field of View Spatial Diagram

Total Variation Models (TVMs) is a classical image restoration method, which is mainly used to recover the real image from the observed image in image processing.

The unconstrained optimization form of the total variation model is:

$$
\min _{u \in X}\left(\|\nabla u\|+\frac{\lambda}{2}\|u-g\|_{2}^{2}\right)
$$

Where $X$ is the vector space of finite dimension, $\nabla$ represents gradient operator, $\lambda$ represents regularization parameter, $u$ represents denoised image, $g$ represents observed image. In the formula, the first term is the regular term, representing the prior information of the original image, and the second term is the fidelity term, which is used to ensure the similarity between the denoised image and the observed image. The regularization parameter is used to balance the regularization term and the fidelity term in the model. When the optimal value is taken, the effect of image restoration is the best. 
Image denoising is an inverse problem of recovering clear image from noisy image. In denoising method, variational method models the inverse problem as an optimization problem of energy functional.

The gradient descent equation of energy functional is

$$
\frac{\partial u}{\partial t}=\operatorname{div}\left(\frac{\nabla u}{|\nabla u|}\right)+\lambda(u-g)
$$

The initial value is set to $u=g$, and the image is iteratively calculated by gradient descent method until an optimal solution is obtained.

$\operatorname{div}\left(\frac{\nabla u}{|\nabla u|}\right)$ is diffusion term. $\frac{1}{|\nabla u|}$ is diffusion coefficient, it is used to balance the diffusion strength of the image under different gradients when repairing the image, so as to avoid over smoothing between the damaged area and its neighborhood after image restoration.

It has the characteristics of a simple model and fewer parameters to be optimized. At the same time, the total variational model has good edge preservation characteristics, which can keep the details of the image while smoothing the image. There are many uncertainties in the field of view, such as illumination brightness, illumination uniformity, smoke and dust in the air. The uncertainty of recognition caused by these environmental variables is eliminated by the total variational model, and accurate image feedback information is obtained.

The process of image information processing: firstly, the image is acquired by the system perception module, then the edge is searched, and the shape recognition, relative position and direction recognition are carried out according to the edge information. Then, the actual position and direction are obtained by the pixel transformation.

Target recognition is essentially a process of finding the data model matching the target from the established 3D data model library. For ease of description, the model base is represented as a set. An element in a set represents a 3D data model, which in turn is represented as a set:

$$
\mathbf{M}_{i}=\left\{F_{k}\right\} \bigcup\left\{V_{j}\right\},(k, j=1,2,3, \ldots)
$$

Formula: a set of geometric primitives constituting the model; a set of model features constructed for estimating pose. The previous pose estimation process is recorded as follows:

$$
P_{n}=f_{e s t}\left(V_{i}, V_{j}\right),\left(V_{j} \in M_{i}\right)
$$

Formula: $P_{n}$-estimated pose. 


\subsection{Ant Colony Algorithm and its optimization}

Assuming that there are $\mathrm{N}_{\mathrm{i}}$ target points in the model base, the probability of ant $\mathrm{K}$ transferring from city $i$ to $j$ is expressed by $\mathrm{P}_{\mathrm{j}}$ at $t$ (city represents each assembly location point in the assembly space):

$$
P_{i j}^{k}(t)=\left\{\begin{array}{l}
\frac{\tau_{i j}^{k}(t) \eta_{i j}^{k}(t)}{\sum_{s_{\text {allowed }}} \tau_{i j}^{\alpha}(t) \eta_{i j}^{\beta}(t)}, j \in \text { allowed }_{k} \\
0, \text { else }
\end{array}\right.
$$

$\eta_{i j}(t)$ is a heuristic factor, indicating the degree of expectation of ants from city $i$ to city $j . \alpha$ and $\beta$ represent the relative importance of pheromones and heuristics, respectively. allowed $_{k}$ is the next step to allow a collection of cities, expressed as

$$
\text { allowed }_{k}=S-\text { tabu }_{k}
$$

$S$ represents the initial point and the set of all target points; $t a b u_{k}$ represents the tabu table of ant $\mathrm{K}$.

To balance the residual pheromones and heuristic information, the following rules are adjusted:

$$
\begin{gathered}
\tau_{i j}(t+n)=\rho \tau_{i j}(t)+\Delta \tau_{i j}(t) \\
\Delta \tau_{i j}(t)=\sum_{k=1}^{m} \Delta \tau_{i j}^{k}(t)
\end{gathered}
$$

In the formula, $\rho$ represents the pheromone residue factor, while $1-\rho$ is the pheromone volatilization factor, $\rho \in(0,1) . \Delta \tau_{i j}(t)$ denotes the pheromone increment on path $(i, j)$ in a cycle, and at the initial time $\Delta \tau_{i j}(0)=0 ; \Delta \tau_{i j}^{k}(t)$ denotes that the ant $\mathrm{K}$ retains the pheromone on path $(i, j)$ in this cycle.

$$
j= \begin{cases}\arg \max _{u \in J(i)}\left\{\tau(i, u)^{\alpha} \eta(i, u)^{\beta}\right\}, & q \leq q_{0} \\ S, & \text { else }\end{cases}
$$

Among them, $\mathrm{q}$ is a random number with uniform distribution between $[0,1] . \mathrm{q}_{0}$ is a parameter $\left(\mathrm{q}_{0} \in[0,1]\right)$, and $S$ is a random variable selected according to the probability distribution given by formula (3). $J(i)$ is an optional collection of cities. $u$ refers to the transferred intermediate City.

The above formula gives a pseudo-random proportional state transition rule. The global pheromone updating rules are executed as follows:

$$
\tau(i, j) \leftarrow(1-\omega) \tau(i, j)+\omega \Delta \tau(i, j)
$$

Global Optimal Path: 


$$
\Delta \tau(i, j)= \begin{cases}\left(L_{g b}\right)^{-1}, & (i, j) \in \text { Global Optimal Path } \\ 0, & \text { else }\end{cases}
$$

Local pheromone updating rules:

$$
\tau(i, j) \leftarrow(1-r) \tau(i, j)+r \Delta \tau(i, j)
$$

When $\Delta \tau(i, j)=\tau_{0} \quad\left(\tau_{0}\right.$ is initial pheromone concentration values on each path), the algorithm can get a better solution in the call time, $r$ is the local pheromone Volatilization Coefficient. The detailed calculation steps are as follows:

Table 1 The detailed calculation steps of the algorithm

\begin{tabular}{ll}
\hline Steps & Content \\
\hline Step 1 & Initializes pheromones and sets other parameters \\
Step 2 & When $d$ is less than $N_{c}$, repeat step 3-step 5. \\
Step 3 & $d=d+1$ \\
Step 4 & $k=k+1$ \\
& Chooses the next target point depending on $P_{i j}^{k}$ and adds the target \\
Step 5 & Point to $S$ and $S$ until ant $\mathrm{K}$ reaches the end point. \\
Step 6 & Updates the pheromone if $k=n_{a}$. \\
Step 7 & Outputs the current optimal solution.
\end{tabular}

At that time (as a constant), the algorithm can be better solved in the call time. The detailed calculation steps are as Table1 $\left(N_{c}\right.$ is Maximum number of iterations, $d$ is Current iterations, $n_{a}$ is Ants number).

When the algorithm falls into the local optimum, the penalty function is introduced to make the pheromone of the current optimal path drop rapidly and reduce the impact of the positive feedback of the next ant search. When the algorithm falls into local optimum, penalty function is introduced $\tau_{\mathrm{ij}}=\lambda \tau_{i j}, \lambda \in(0,1)$.

1) In the process of ant search, when encountering complex terrain, it is easy to form path deadlock. In order to make the pheromone on the path around the trap drop rapidly without affecting the next ant search, a penalty function is introduced.

2) The length of the optimal path has not changed for 20 consecutive generations, and the penalty function is introduced. An improved pheromone updating formula:

$$
\tau_{\mathrm{ij}}=\left\{\begin{array}{l}
\lambda \tau_{i j}, \text { if }(\mathrm{i}, \mathrm{j}) \in \text { Global Optimal Path } \\
\tau_{i j}, \text { else }
\end{array}\right.
$$

\subsection{General Process of Path Planning}

The path planning of assembly process execution mechanism refers to the process that fixtures, work pieces, and tools are located and generated by visual methods in the assembly process, and the generated path is optimized. 
The main steps are as Table 2:

Table 2 The main steps of path planning

\begin{tabular}{ll}
\hline Step 1 & $\begin{array}{l}\text { Obtains and processes the field of view spatial information (unit information } \\
e \text {, spatial type } S \text {, topological structure } T \text { ); }\end{array}$ \\
\hline Step 2 & $\begin{array}{l}\text { Generates assembly path } P_{0} \text { according to the result of FOV spatial } \\
\text { information processing } \\
\text { Judges whether the generated path collides or not, and if there is collision (the } \\
\text { estimated pose and path } P_{0} \text { intersect), the collision analysis is carried out by } \\
\text { the system perception module, and then returns to } 1 \text {; if there is no collision, go } \\
\text { to } 4 \text {; }\end{array}$ \\
Step 3 & $\begin{array}{l}\text { Determines whether or not the target points } k_{1}, k_{2}, k_{3}, \ldots k_{n} \text { are reached, if } \\
\text { not, the online path optimization is carried out, and then returns to } 2 \text {; if the } \\
\text { target point is reached, it goes to 5; }\end{array}$ \\
Step 5 & $\begin{array}{l}\text { Moves the actuator according to the non-collision path. } \\
\text { Saves all points on the path according to the motion information of the } \\
\text { actuator. }\end{array}$ \\
Step 7 & $\begin{array}{l}\text { Judges whether the assembly is complete or not, and returns to } 1 \text { if the } \\
\text { assembly is not completed; if the assembly is completed, the process is } \\
\text { completed. }\end{array}$ \\
\hline
\end{tabular}

\section{Examples of Verification}

\subsection{Background and Data collection}

Data acquisition is very important for evaluation and improvement. The evaluation of the path planning algorithm should include the length of the path and the time consumed. These basic data should include the length and time of path planning in a static environment and the length and time of path optimization in a dynamic environment.

Table 3 is the assembly process flow of upper and lower valve bodies, including locating pin, bushing, valve element, installation spring, solenoid valve, installation of upper and lower valve bodies. There are 17 stations from huc-010 to huc170.

Table 3 Assembly process of upper and lower valve bodies

\begin{tabular}{ll}
\hline Station number & Work content \\
\hline HCU-010 & Install the locating pin \\
HCU-020 & Install the lower body bushing and valve element \\
HCU-030 & Install spring \\
HCU-040 & Test spring force \\
HCU-050 & Install O-ring and blank \\
HCU-060 & Install the upper body solenoid \\
HCU-070 & Install the right solenoid ball and spring \\
HCU-080 & Install the upper body bushing and valve element \\
HCU-090 & Test spring force \\
HCU-100 & Install the upper body solenoid \\
HCU-110 & Install the left solenoid ball and spring
\end{tabular}


HCU-120

HCU-130

HCU-140

HCU-150

HCU-160

HCU-170
Install the accumulator spring, piston and upper and lower plates Install 21 connecting bolts of clutch pressure sensor and valve body Install 9 bolts connecting the valve body and pre-tighten them Tighten bolts Install the harness bracket and tighten 6 bolts Insert the wire harness of solenoid valve into the plug of solenoid valve and clutch sensor, recheck the state of engagement

After assembly, as shown in Figure 3:

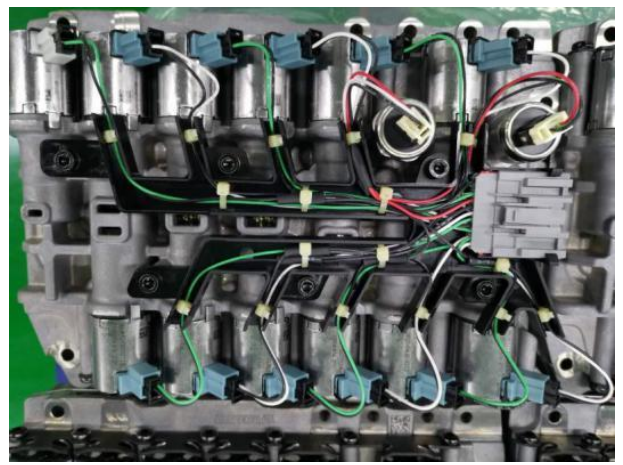

Fig 3 Assembled upper valve body

In line with the tide of industrial 4.0, automobile manufacturing has begun to move towards digitalization, automation, and intellectualization. Engines and gearboxes are the most important power parts of automobiles, and the quality of their processing and assembly is related to the quality of the whole vehicle. Our team participated in data acquisition and visualization of DCT dual-clutch transmission of Jianghuai Automobile Co. Ltd(JAC). This paper will take the assembly of the valve plate on the solenoid valve of DCT dual-clutch transmission of JAC as an example to verify the assembly path planning. It is verified in this paper that 9 bolts are pre-tightened for huc- 140 valve body.

In this example, applying the concept of field space, the work piece type is the valve body of DCT gearbox solenoid valve, the number of different valve body target points may be 9,12 or 7 , the position of obstacles may be blocked by the position of harness and bracket, and the position of cage may be blocked.

In the case of map in this case, when other parameters remain unchanged, several groups of $\alpha$ and $\beta$ are selected for comparison. It is found that when $\alpha=1 / 2, \beta=5$, the distance average value and standard deviation of the optimal path are the smallest, and the average time is small.

Parameter settings are shown in Table 4. 
Table 4 Parameter settings

\begin{tabular}{lcccc}
\hline \multicolumn{1}{c}{ parameter } & ACS algorithm & $\begin{array}{c}\text { Control } \\
\text { group 2 }\end{array}$ & $\begin{array}{c}\text { Control } \\
\text { group 3 }\end{array}$ & This paper \\
\hline Number of ants & 20 & 50 & 25 & 25 \\
Heuristic factor & 1 & 0.5 & 1 & 0.5 \\
Expectation heuristics & 2 & 5 & 5 & 5 \\
Selection threshold & 0.8 & 0.8 & 0.8 & 0.8 \\
Volatilization Coefficient of & 0.1 & 0.1 & 0.1 & 0.1 \\
local pheromone & 0.1 & 0.1 & 0.1 & 0.1 \\
Global pheromone volatility & 200 & 200 & 200 & 200 \\
Maximum number of & & & & \\
iterations & & &
\end{tabular}

\subsection{Examples to verify ideas}

According to the detailed description of the assembly environment in the construction diagram, this paper uses the system model shown in Figure 4 to study the path planning of a single manipulator in the assembly environment. Line body, manipulator, fixture and valve body constitute assembly space. Guppy camera monitors obstacles and environmental changes. FANUC robot and fixture are used to assemble valve plate bolts on solenoid valves in static and dynamic environments respectively.
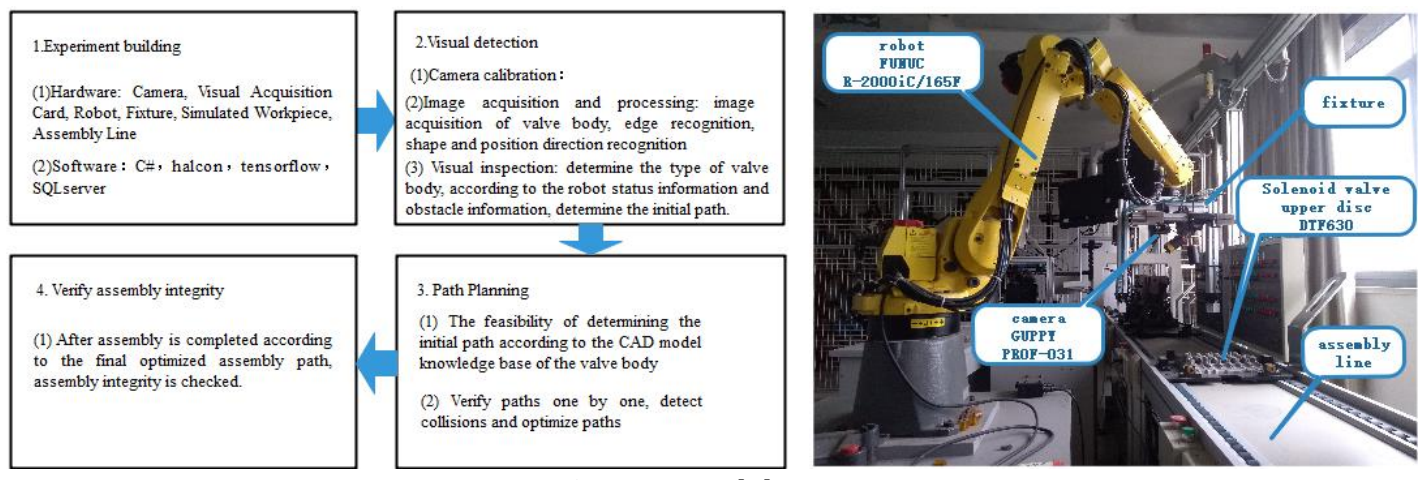

Fig 4 System Model Diagram

\subsection{Results}

To verify the performance of the algorithm in solving the on-line path planning of a single manipulator, the valve plates on solenoid valves are assembled and tested in static and dynamic environments respectively. Compare the length of route planning optimization under static conditions and the dynamic response time of obstacle avoidance. 


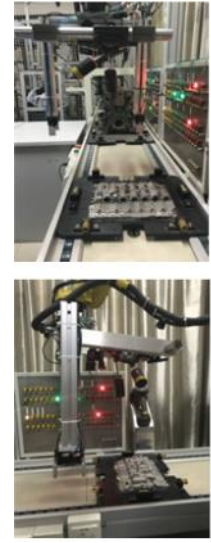

a) Visual layout
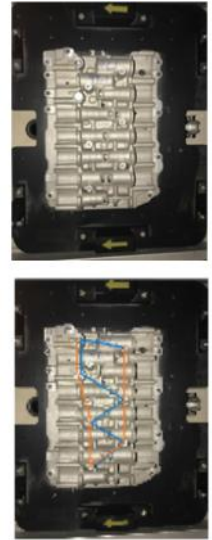

b) Path planning results

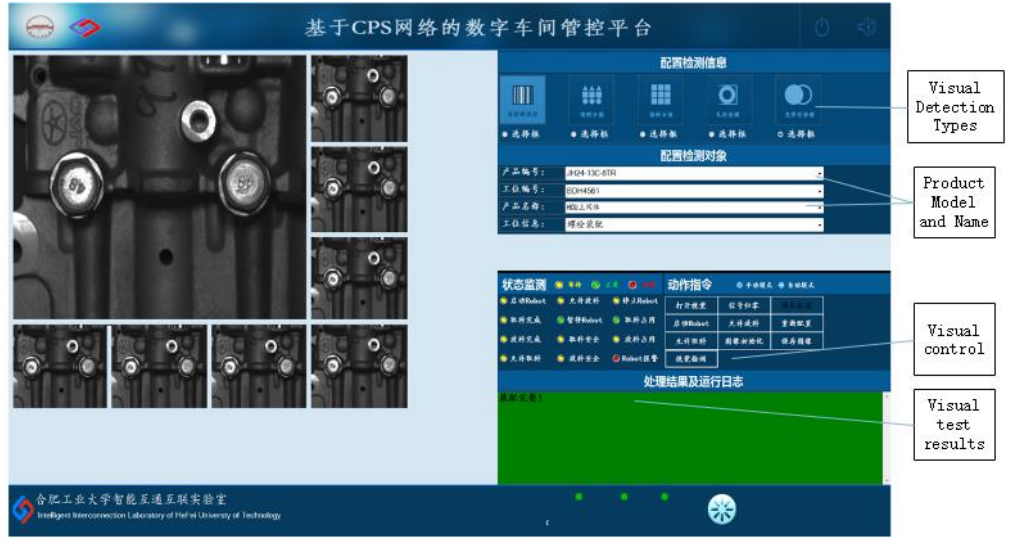

c) Assembling Integrity Testing

Fig 5 Assembly Path Planning of Engine Top Cover Bolts

From Figure 5, we can see that the target part carries out path planning by finding feasible grids outside obstacles, in which the blue line is the initial path planning and the red line is the optimized path.

20 groups of valve bodies are selected for testing, including 7 of 7 target points, 7 of 9 target points and 6 of 12 target points. There are three initial states of the valve body of the three target points, which are normal state, left offset state and again offset state. 20 groups of path planning were carried out for static and dynamic executing agencies respectively.

Dynamic situation: when the value body target points change in 9, 12 and 7, and there are red obstacles in the figure, the system can give corresponding judgment and adjust the planning path in real time.

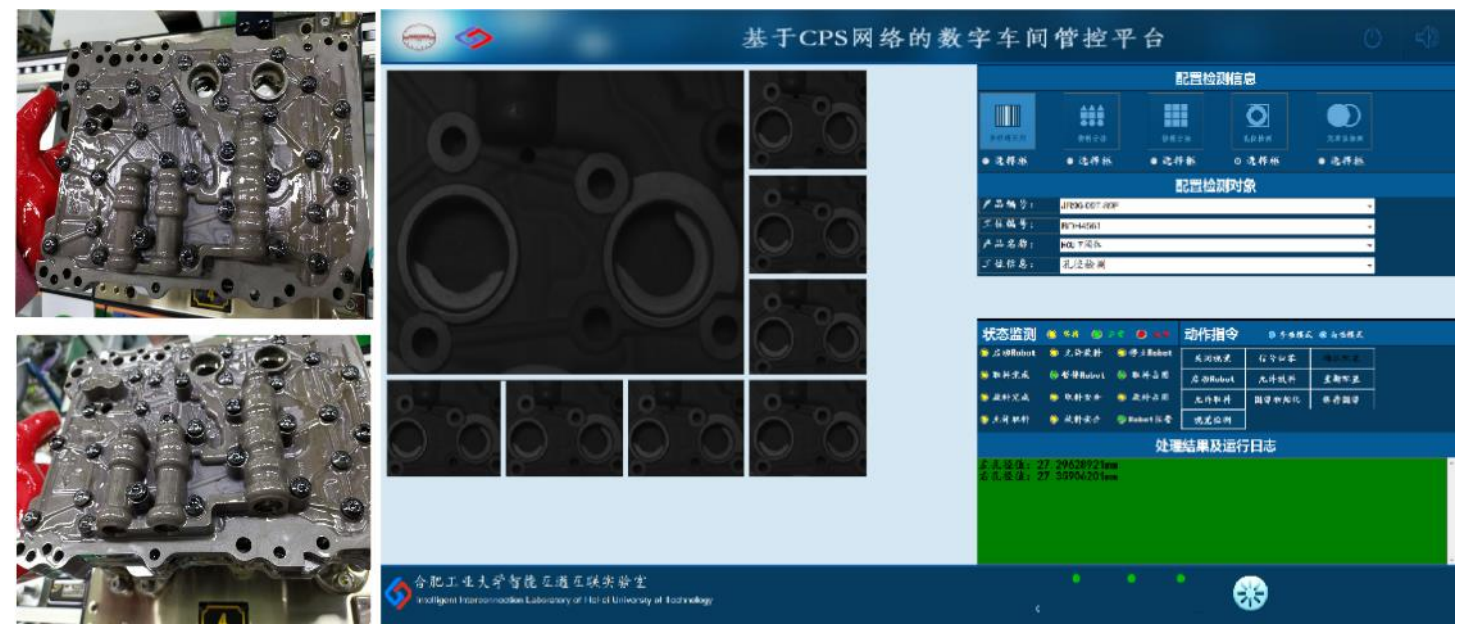

Fig 6 Dynamic scene space and assembly path planning

Table 5 shows that the ant colony algorithm can quickly find the initial assembly path of parts in complex environment. The whole calculation process is $0.2800-0.7000 \mathrm{~s}$. The ant colony algorithm has the advantages of swarm intelligence and so on. It has high solving efficiency in path planning. 
Table 5 Assembly path planning and optimization case results

\begin{tabular}{lcc}
\hline Bolt assembly of engine top cover & Static state & Dynamic state \\
\hline Initial average time (s) & 0.2800 & 0.7000 \\
Optimizing Average Time (s) & 0.0025 & 0.0045 \\
Initial path length (mm) & 875.59 & 725.36 \\
Optimizing path length (mm) & 664.83 & 702.59 \\
\hline
\end{tabular}

Figure 7 shows the curve of the shortest path length with the number of iterations of ACS algorithm and the algorithm in this paper in the obstacle avoidance environment.

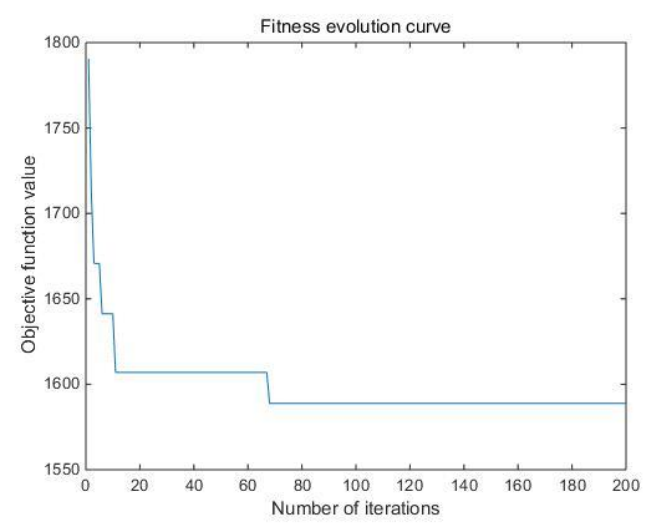

a

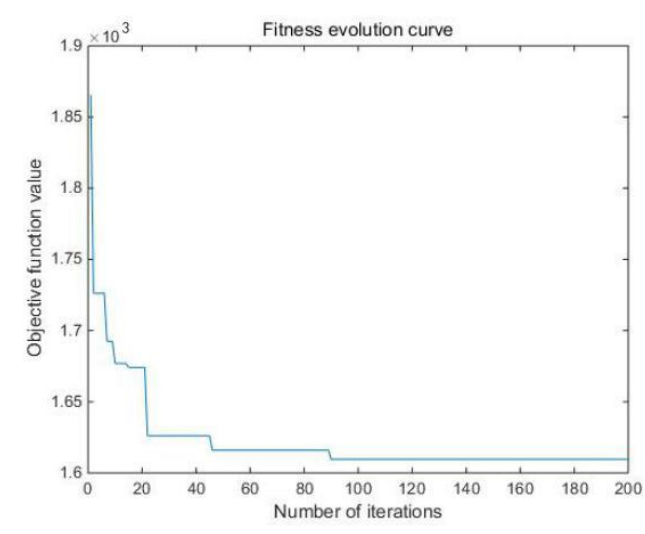

c

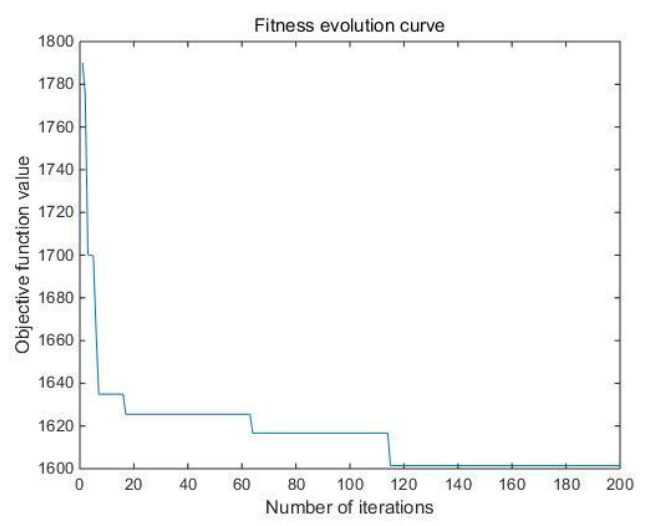

b

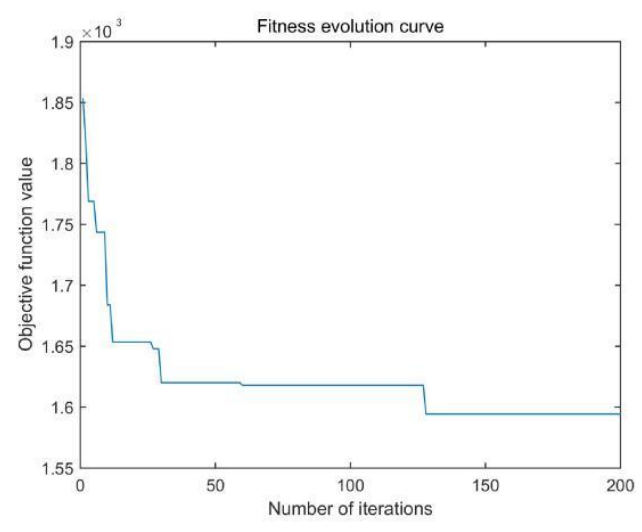

$\mathrm{d}$

Fig 7 Curve of shortest path length of algorithm in this paper(a), ACS algorithm(b), control group 2(c) and control group 3(d)

It can be seen from figure 7 that the iterations of ACS algorithm and this algorithm are 117 and 63 respectively, so the convergence speed of this algorithm is faster than that of ACS algorithm, control group 2 and control group 3, and the final shortest path length of convergence is better than ACS algorithm, control group 2 and control group 3.

As shown in Table 6. Compared with Ant Colony Algorithm, Genetic Algorithm has faster convergence speed but longer optimal path. The optimal path of Simulated Annealing Algorithm is almost the same, but its convergence speed is slow. 
Table 6 Compared with Genetic Algorithm and Simulated Annealing Algorithm when target points are 12

\begin{tabular}{lcc}
\hline algorithm & Shortest path length(mm) & $\begin{array}{c}\text { Convergence average search } \\
\text { time(ms) }\end{array}$ \\
\hline method of this paper & 1588 & 907 \\
Genetic Algorithm & 1644 & 611 \\
Simulated Annealing & 1612 & 1490 \\
Algorithm & & \\
\hline
\end{tabular}

As shown in Table 7. The comparison of simulation data under the three conditions of 7, 9 and 12 target points is given. It can be seen that the number of convergence iterations, iteration time and shortest path length of this algorithm are less than those of ACS algorithm, control group 2 and control group 3.

Table 7 Comparison of simulation data of four algorithms

\begin{tabular}{ccccccccccccc}
\hline \multirow{2}{*}{$\begin{array}{c}\text { target } \\
\text { points }\end{array}$} & \multicolumn{1}{c}{ Shortest path length(mm) } & \multicolumn{4}{c}{ Convergence average } & \multicolumn{4}{c}{ Convergence average search } \\
& $1^{*}$ & 2 & 3 & 4 & 1 & 2 & 3 & 4 & 1 & 2 & 3 & 4 \\
\hline 7 & 830 & 850 & 780 & 702 & 15 & 17 & 16 & 11 & 230 & 244 & 169 & 134 \\
9 & 1100 & 1122 & 1109 & 1025 & 70 & 80 & 66 & 38 & 570 & 583 & 431 & 290 \\
12 & 1607 & 1611 & 1595 & 1588 & 117 & 90 & 133 & 63 & 1442 & 1459 & 1223 & 907 \\
\hline *1 represent ACS algorithm, 2 represent control group 2 in Table 2, 3 represent control group 3 in \\
Table 2, 4 represent method of this paper.
\end{tabular}

\section{Conclusion}

The accuracy and efficiency of mechanical manufacturing process is the focus of manufacturing process. Monitoring and adjusting the manufacturing process through machine vision technology can improve the accuracy and efficiency. The adjustment of machine vision technology is a necessary way to promote the optimization of manufacturing process

In this paper, the single manipulator operation in assembly process is taken as the research background. Considering the equipment resources, the assembly sequence and assembly path planning of products are studied. The product information model based on FOVS is established, and the assembly sequence generation algorithm considering equipment resource information is given. The simulation of assembly planning structure shows that the result of assembly planning is better than ACS algorithm. The product information model in view space is established, the data structure of the product model is analyzed, and the part information model including assembly features is established, which realizes the unified expression of geometric data information, expresses the dynamic constraints of parts and their assembly resources in the assembly process, and reduces the complexity of the path planning of single manipulator in the assembly process. 
The online path planning method of assembly robots based on FOVS can further agglomerate and strengthen the core competitiveness and improve the improve the accuracy and efficiency of manufacturing process. It can be applied to any situation in the manufacturing process, not just assembly robots. Its disadvantage is that it does not consider the dynamic change, or the external factors of industrial production system, which is the next research direction.

\section{Declarations}

\section{Ethical approval}

Ethical approval was not required for this study.

\section{Consent to participate}

Written informed consent was obtained from individual or guardian participants.

\section{Consent to publish}

Manuscript was approved by all authors for publication.

\section{Authors contributions}

Mingzhou Liu: Investigation, methodology, writing original draft. Xin Xu: Visualization, project administration, resources. Xiaoqiao Wang: Supervision, and validation. Maogen Ge: Data curation, software. Lin Ling: Conceptualization, Experiment. Conghu Liu: Writing review, and editing.

\section{Funding}

Funding information is not applicable.

\section{Competing interests}

The authors declare that they have no known competing financial interests or personal relationships that could have appeared to influence the work reported in this paper.

\section{Availability of data and materials}

The datasets used or analyzed during the current study are available from the corresponding author on reasonable request. 


\section{References}

[1] He Y, Yu Z, Li J, et al. Weld seam profile extraction using top-down visual attention and fault detection and diagnosis via EWMA for the stable robotic welding process[J]. The International Journal of Advanced Manufacturing Technology, 2019, 104(9-12):3883-3897.

[2] Dong G, Marleau-Finley J, Zhao Y F. Investigation of electrochemical post-processing procedure for Ti-6Al-4V lattice structure manufactured by direct metal laser sintering (DMLS)[J]. International Journal of Advanced Manufacturing Technology, 2019, 104(10).

[3] Liu Z, Wang Y, Wu B, et al. A critical review of fused deposition modeling 3D printing technology in manufacturing polylactic acid parts[J]. The International Journal of Advanced Manufacturing Technology, 2019.

[4] Nerakae P., Uangpairoj P., Chamniprasart K (2016). Using Machine Vision for Flexible Automatic Assembly System. Elsevier Science Publishers B. V.

[5] Liu C., Zhu Q., Wei F., et al. (2019) An integrated optimization control method for remanufacturing assembly system. Journal of Cleaner Production,119-261

[6] He, KT., Zhang, Q., Hong, YL. (2019). Profile monitoring based quality control method for fused deposition modeling process. Journal of Intelligent Manufacturing. 30(2), 947-958.

[7] Miguel, A., Simao, Olivier, Gibaru. (2019). Online Recognition of Incomplete Gesture Data to Interface Collaborative Robots. IEEE Transactions on Industrial Electronics. 66(12), 9372-9382.

[8] Xianjie, Pua., Hengyu, Guoa., Qian Tang. (2018). Rotation sensing and gesture control of a robot joint via triboelectric quantization sensor. NANO Energy. 54, 453-460.

[9] Mohsen, Kaboli., Kunpeng, Yao., Di Feng. (2018). Tactile-based active object discrimination and target object search in an unknown workspace. Autonomous Robots. 43, 123-152.

[10] Behnoosh, Parsa., Ekta, U., Samani., Rose, Hendrix. (2019). Toward Ergonomic Risk Prediction via Segmentation of Indoor Object Manipulation Actions Using Spatiotemporal Convolutional Networks. IEEE Robotics and Automation Letters. 4(4), 3153-3160.

[11] Chenghao, Bi., Maria, Guix., Benjamin, V. Johnson. (2018). Design of Microscale Magnetic Tumbling Robots for Locomotion in Multiple Environments and Complex Terrains. MicroMachines. 9(2), 1-17.

[12] Adel, Bakhshipoura., Abdolabbas, Jafari. (2018). Evaluation of support vector machine and artificial neural networks in weed detection using shape features. Computers and Electronics in Agriculture. 145, 153-160.

[13] Chenming, Wu., Rui, Zeng., Jia, Pan., Charlie, C. L. Wang. (2019). Plant Phenotyping by Deep-Learning-Based Planner for Multi-Robots. IEEE Robotics and Automation Letters. 4(4), 3113-3120.

[14] Yao, Guo., Fani, Deligianni., Xiao, Gu. (2019). 3-D Canonical Pose Estimation and Abnormal Gait Recognition with a Single RGB-D Camera. IEEE Robotics and Automation Letters. 4(4), 3617-3624.

[15] Noriaki, Hirose., Fei, Xia., Roberto, Martín-Martín. (2019). Deep Visual MPC-Policy Learning for Navigation. IEEE Robotics and Automation Letters. 4(4), 3184-3191.

[16] Kaiming, He., Xiangyu, Zhang., Shaoqing, Ren. (2015). Delving Deep into Rectifiers: Surpassing Human-Level Performance on ImageNet Classification. arXiv:1502.01852v1 [cs.CV]

[17] Shaoqing, Ren., Kaiming, He., Ross, Girshick. (2017). Faster R-CNN: Towards Real-Time ObjectDetection with Region Proposal Networks. IEEE Transactions on Pattern Analysis and Machine Intelligence. 39(6), 1137-1149.

[18] Kaiming, He., Jian, Sun., Xiaoou, Tang. (2011). Single Image Haze Removal Using Dark Channel Prior. IEEE Transactions on Pattern Analysis and Machine Intelligence. 33(12), 2341-2353.

[19] Chao, Dong., Chen, Change, Loy., Kaiming, He. (2016). Image Super-Resolution Using Deep Convolutional Networks. IEEE Transactions on Pattern Analysis and Machine Intelligence. 38(2), 295-307.

[20] Kaiming, He., Xiangyu, Zhang., Shaoqing, Ren. (2016). Deep Residual Learning for Image Recognition. IEEE Conference on Computer Vision and Pattern Recognition. 10.1109, 770-778.

[21] C. Lawrence, Zitnick., Piotr, Dollar. (2014). Edge Boxes: Locating Object Proposals from Edges. Computer Vision. 8693, 391-405.

[22] Piotr, Dollar., Christian, Wojek., Bernt, Schiele. (2012). Pedestrian Detection: An Evaluation of the State of the Art. IEEE Transactions on Pattern Analysis and Machine Intelligence. 34(2), 743-761.

[23] Piotr, Dollar., C. Lawrence, Zitnick. (2014). Microsoft Research Fast Edge Detection Using Structured Forests. IEEE Transactions on Pattern Analysis and Machine Intelligence. 37(8), 1558-1570.

[24] Wei, Liu., Xianqiao, Chen., Xiumin, Chu. (2016). Haze removal for a single inland waterway image using sky segmentation and dark channel prior. IET Image Processing. 10(12), 996-1006.

[25] Evan, Shelhamer., Jonathan, Long., Trevor, Darrell. (2017). Fully Convolutional Networks for Semantic Segmentation. IEEE Transactions on Pattern Analysis and Machine Intelligence. 39(4), 640-651.

[26] Lei, Yan., Wenfu, Xu., Zhonghua, Hu. (2019). Virtual-base modeling and coordinated control of a dual-arm space robot for target capturing and manipulation. Multibody System Dynamics. 45(4), 431-455.

[27] Zhou, Xian., Puttichai, Lertkultanon., Quang-Cuong, Pham. (2017). Closed-Chain Manipulation of Large Objects by Multi-Arm Robotic Systems. IEEE Robotics and Automation Letters. Preprint Version 10(1109), 1-8.

[28] Matko, Orsag., Christopher, Korpela., Stjepan, Bogdan. (2014). Valve Turning using a Dual-Arm Aerial 
Manipulator. International Conference On Unmanned Aircraft Systems. 836-841.

[29] Sergio, Cubero., Nuria, Aleixos., Enrique, Moltó. (2011). Advances in Machine Vision Applications for Automatic Inspection and Quality Evaluation of Fruits and Vegetables. Food Bioprocess Technol. 4, 487-504.

[30] Aihui, Wang., Mingcong, Deng. (2012). Operator-based Robust Nonlinear Tracking Control for A Human Multi-Joint Arm-like Manipulator with Unknown Time-varying Delays. Applied Mathematics \& Information Sciences. 6(3), 459-468.

[31] Lingling, Shi., Hiranya, Jayakoby., Jayantha, Katupitiya. (2018). Coordinated Control of a Dual-Arm Space Robot. IEEE Robotics \& Automation Magazine. 25(4), 86-95.

[32] Qingsong, Xu. (2017). Design and Development of a Novel Compliant Gripper with Integrated Position and Grasping/Interaction Force Sensing. IEEE Transactions on Automation Science and Engineering. $14(3), 1415-1428$.

[33] Anouar, Benamor., Wafa, Boukadida., Hassani, Messaoud. (2019). Genetic algorithm-based multi-objective design of optimal discrete sliding mode approach for trajectory tracking of nonlinear systems. Proceedings of The Institution of Mechanical Engineers Part C-Journal of Mechanical Engineering Science. 233(15), 5237-5252.

[34] Yue, Wang., Yanmei, Jiao., Rong, Xiong. (2018). MASD: A Multimodal Assembly Skill Decoding System for Robot Programming by Demonstration. IEEE Transactions on Automation Science and Engineering. 15(4), 1722-1734.

[35] Wafa, Boukadida., Anouar, Benamor. (2019). Multi-Objective Design of Optimal Sliding Mode Control for Trajectory Tracking of SCARA Robot Based on Genetic Algorithm. Journal of Dynamic Systems Measurement and Control-Transactions of The Asme. 141(3), 031015 1-11.

[36] Seyed, Mohammad, Ahmadi., Mohammad, Mehdi, Fateh. (2019). Task-space control of robots using an adaptive Taylor series uncertainty estimator. International Journal of Control. 92(9), 2159-2169.

[37] Phung, M, D., Quach, C, H., Dinh, T, H., et al. (2017)Enhanced discrete particle swarm optimization path Xue Juntao; Xu Shaopeng; Wang Ye.(201)planning for UAV vision-based surface inspection. Automation in Construction, 81,25-33.

[38] Xue, Juntao., Xu, Shaopeng., Wang, Ye.(2016)Path planning of intelligent car based on computer vision.Computer Engineering and Applications.52(7),236-241,247.

[39] Qizhi, Chen., Chengrui, Zhang., Hepeng, Ni. (2018). Trajectory planning method of robot sorting system based on S-shaped acceleration/deceleration algorithm. International Journal of Advanced Robotic Systems. 15(6), 1-13.

[40] Syafrudin, Muhammad., Alfian, Ganjar.(2018)Performance Analysis of IoT-Based Sensor, Big Data Processing, and Machine Learning Model for Real-Time Monitoring System in Automotive Manufacturing.18(9),1424-8220.

[41] Chen, M., Xin, D., Woods, D. (2015) Parallel computation using active self-assembly. Natural Computing, $14(2), 225-250$

[42] Roussel, R., Cani, M, P., Jean-Claude, Léon., et al. (2018) Exploratory design of mechanical devices with motion constraints. Computers \& Graphics, 74(AUG.), 244-256.

[43] Pellegrinelli, S., Pedrocchi, N. (2017) Estimation of robot execution time for close proximity human-robot collaboration. Integrated Computer-Aided Engineering, 25(1),81-96.

[44] Baynal, K., Sari, T., Akpinar, B.(2018)Risk management in automotive manufacturing process based on FMEA and grey relational analysis: A case study. Advances in Production Engineering \& Management.13(1), 69-80.

[45] Sikdar, S., Kudela, P., Radzienski, M., et al. (2018) Online detection of barely visible low-speed impact damage in 3D-core sandwich composite structure. Composite Structures, 185,646-655.

[46] Oztemel E., Gursev S (2018). Literature review of Industry 4.0 and related technologies. Journal of Intelligent Manufacturing, 1-56.

[47] Tamang, P., \& Kumar, P. (2015). A DBMS based inventory model and its timeframe study in automobile spare parts import management. In 9th International conference on software, knowledge, information management and applications , $321-328$

[48] E S Soegoto., R D Utami and Y A Hermawan (2019). Influence of artificial intelligence in automotive industry. Journal of Physics: Conference Series.

[49] Liu, GQ., Gao, XD., You, DY., Zhang, NF. (2019). Prediction of high-power laser welding status based on PCA and SVM classification of multiple sensors. Journal of Intelligent Manufacturing. 30(2). 821-832.

[50] Keyu, Wu., Mahdi, Abolfazli, Esfahani., Shenghai, Yuan. (2019). TDPP-Net: Achieving three-dimensional path planning via a deep neural network architecture. Neurocomputing. 357, 151-162.

[51] MH, Korayem., R, Abbasi Esfeden., SR, Nekoo. (2015). Path planning algorithm in wheeled mobile manipulators based on motion of arms. Journal of Mechanical Science and Technology. 29(4), 1753-1763.

[51] Sotiris, Makris., Panagiota, Tsarouchi. (2017). Dual arm robot in cooperation with humans for flexible assembly. CIRP Annals - Manufacturing Technology. 66, 13-16. 
Figures
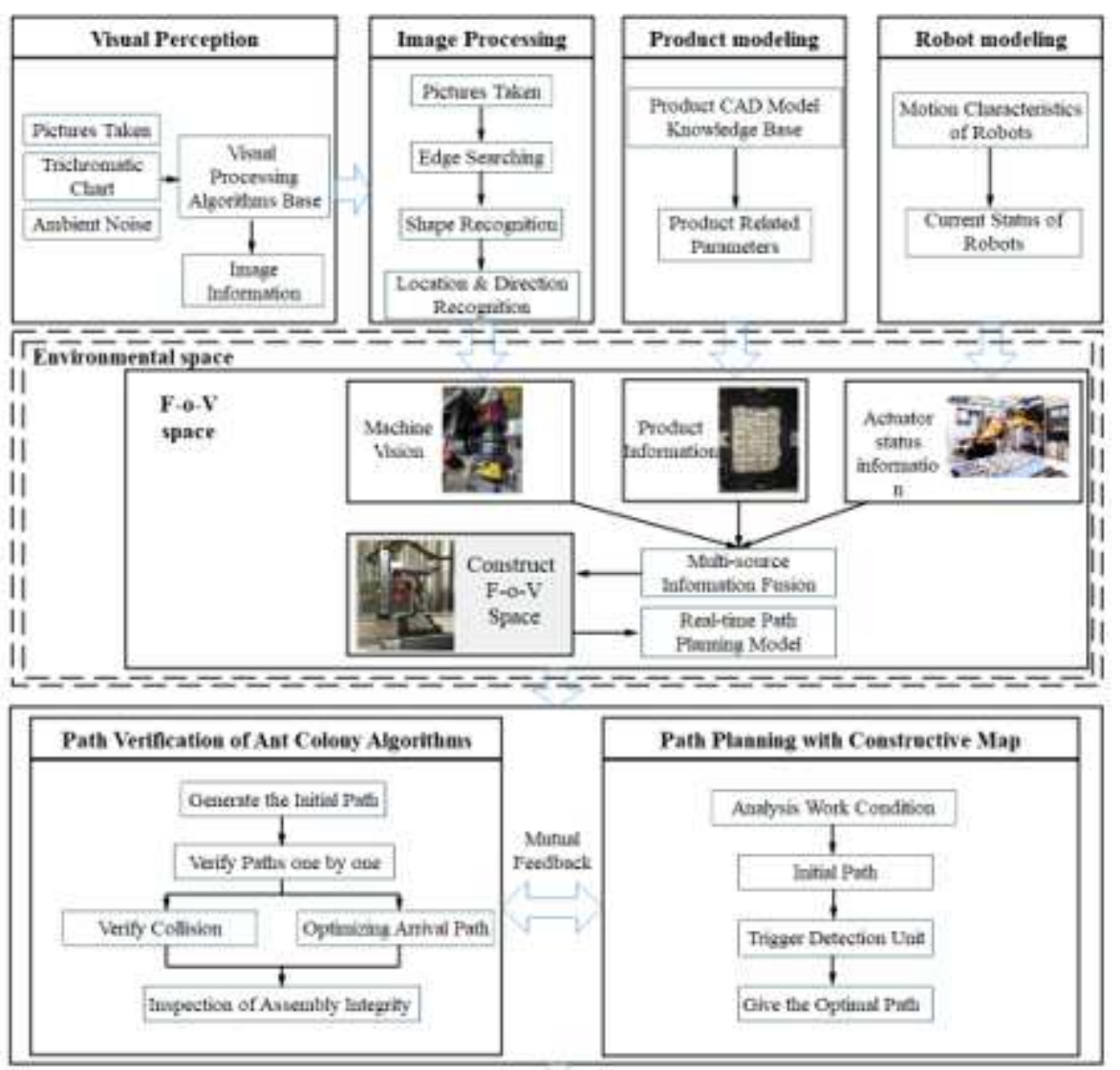

Optimize and Cornplete Assembly, and Give

Optimal Path Length and Optimal Time

\section{Figure 1}

general framework 


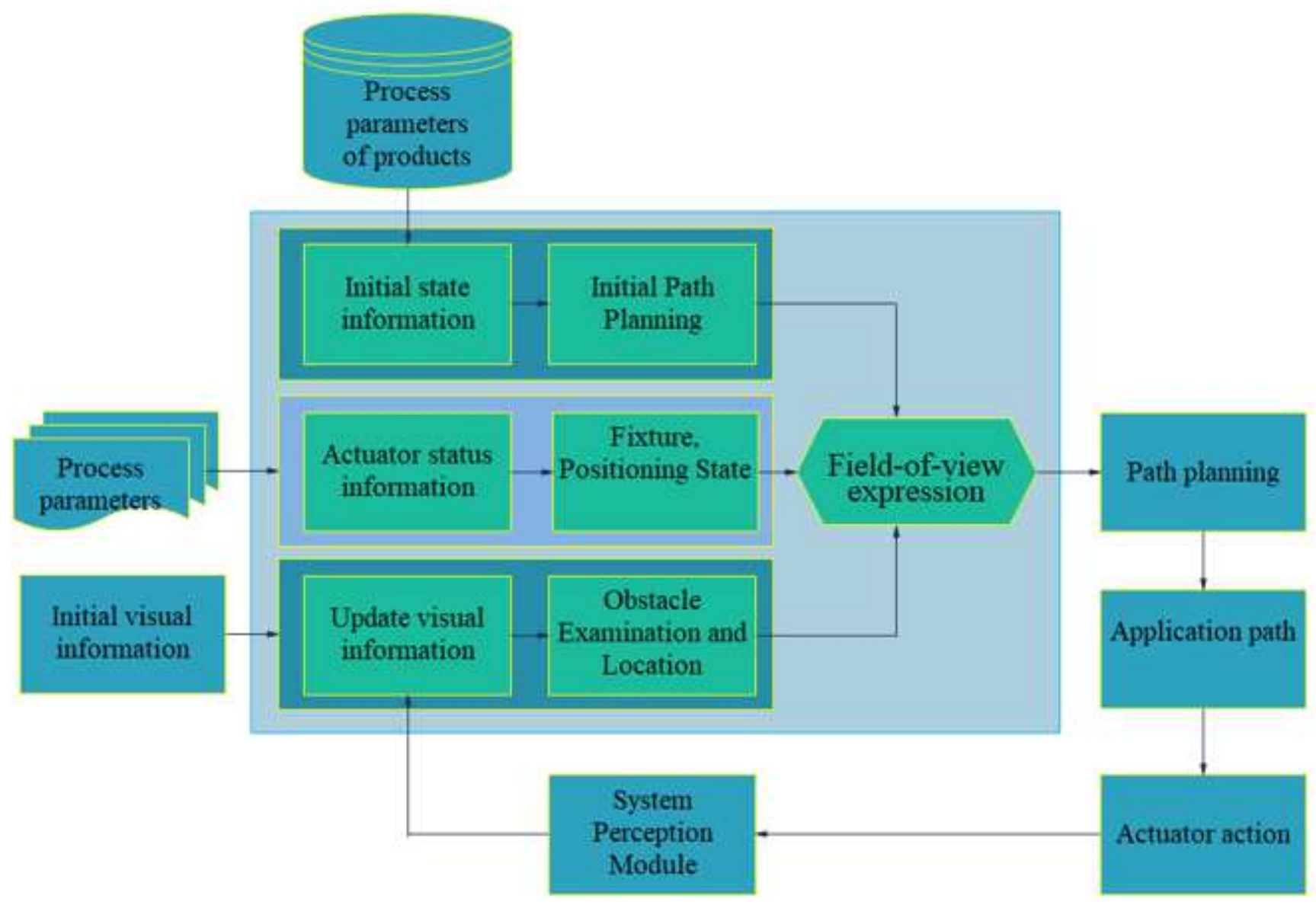

Figure 2

Field of View Spatial Diagram

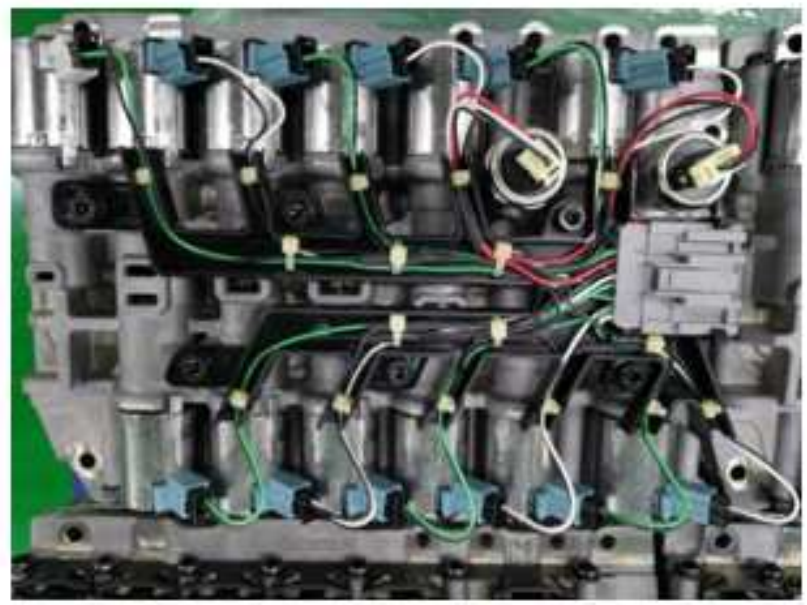

Figure 3

Assembled upper valve body 


1Experiment building
(1)Hardware: Camera, Visual Acquistion
Card, Robot, Future, Simulated Wotlpiece,
Assembly Line
(2)Softwate 2 Cr, halcon * tens orflow,
SQL server

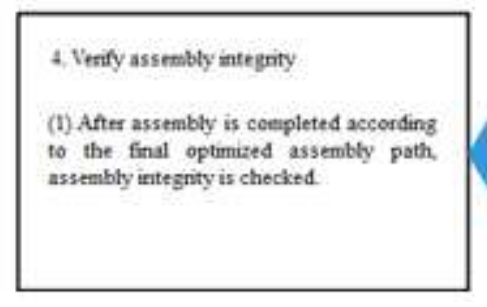

2.Visual detection

(1)Canera callbation:

(2) Image acquisition and processing: image acquisition of valve body, edge tecognition. shape and position drection recognition

(5) Visual inspection: determine the type of valve body, according to the robot status information and obstacle infoemation, detemine the initial path

\section{$+2$}

\section{Path Planing}

(1) The feasibility of deternining the initial path accerding to the $\mathrm{CAD}$ miodel lnouledge base of the valve body.

(2) Venfy paths one by one, detect collisions and optimize paths

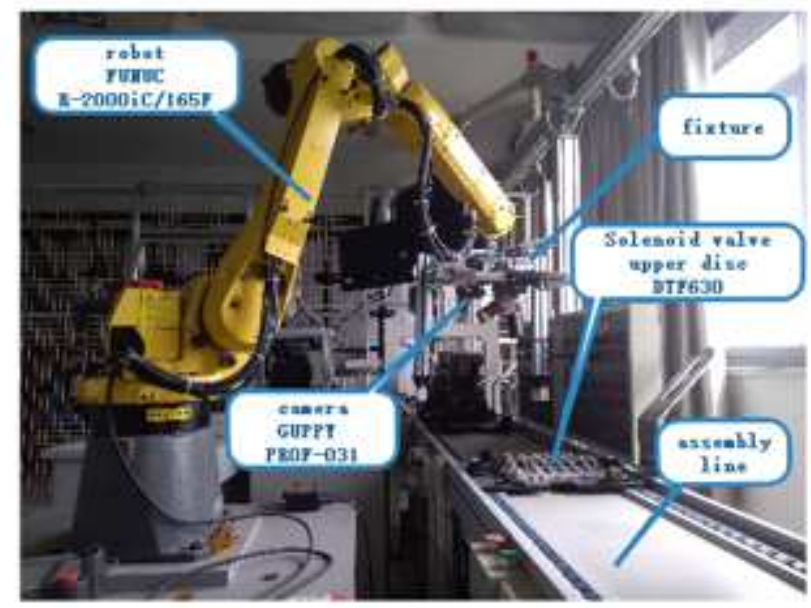

Figure 4

System Model Diagram

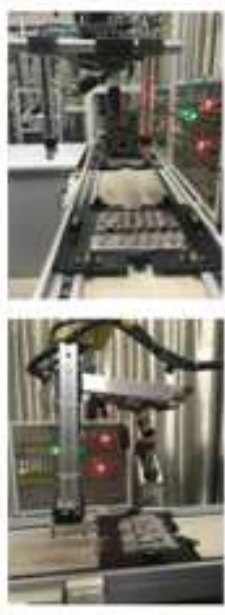

a) Visual layout
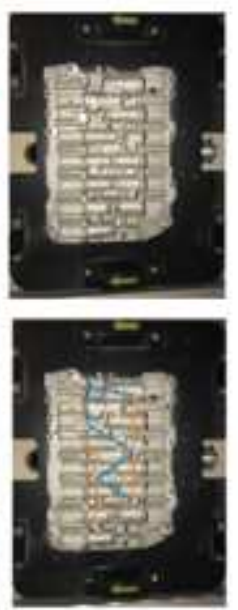

b) Path planning results

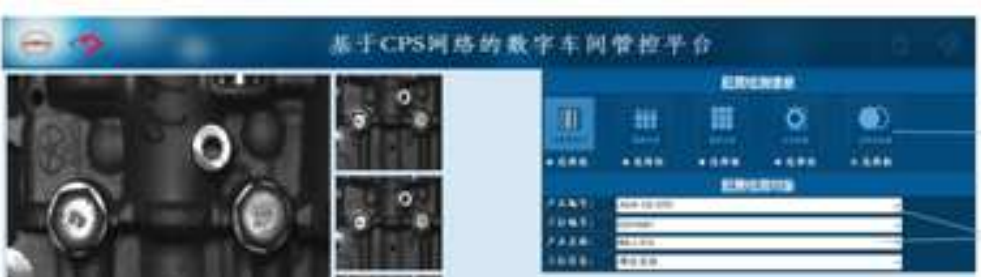

Visual

Detectian

Fobuct
Hode1

and Hine
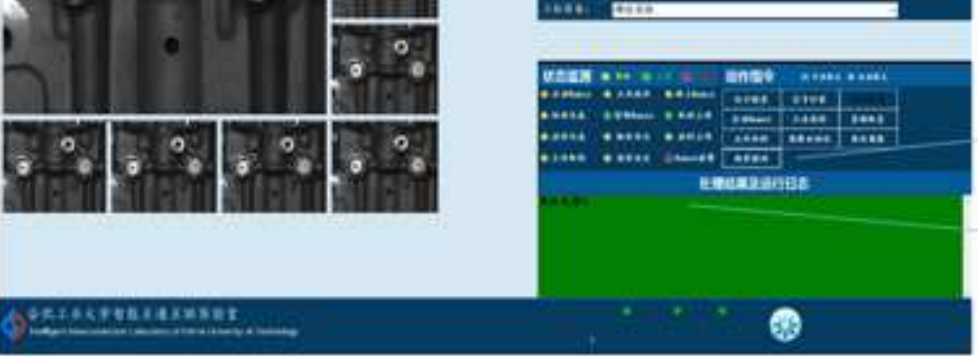

Viras. cient $x$ al Vimal teralt

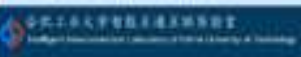

c) Assembling Integrity Testing

\section{Figure 5}

Assembly Path Planning of Engine Top Cover Bolts

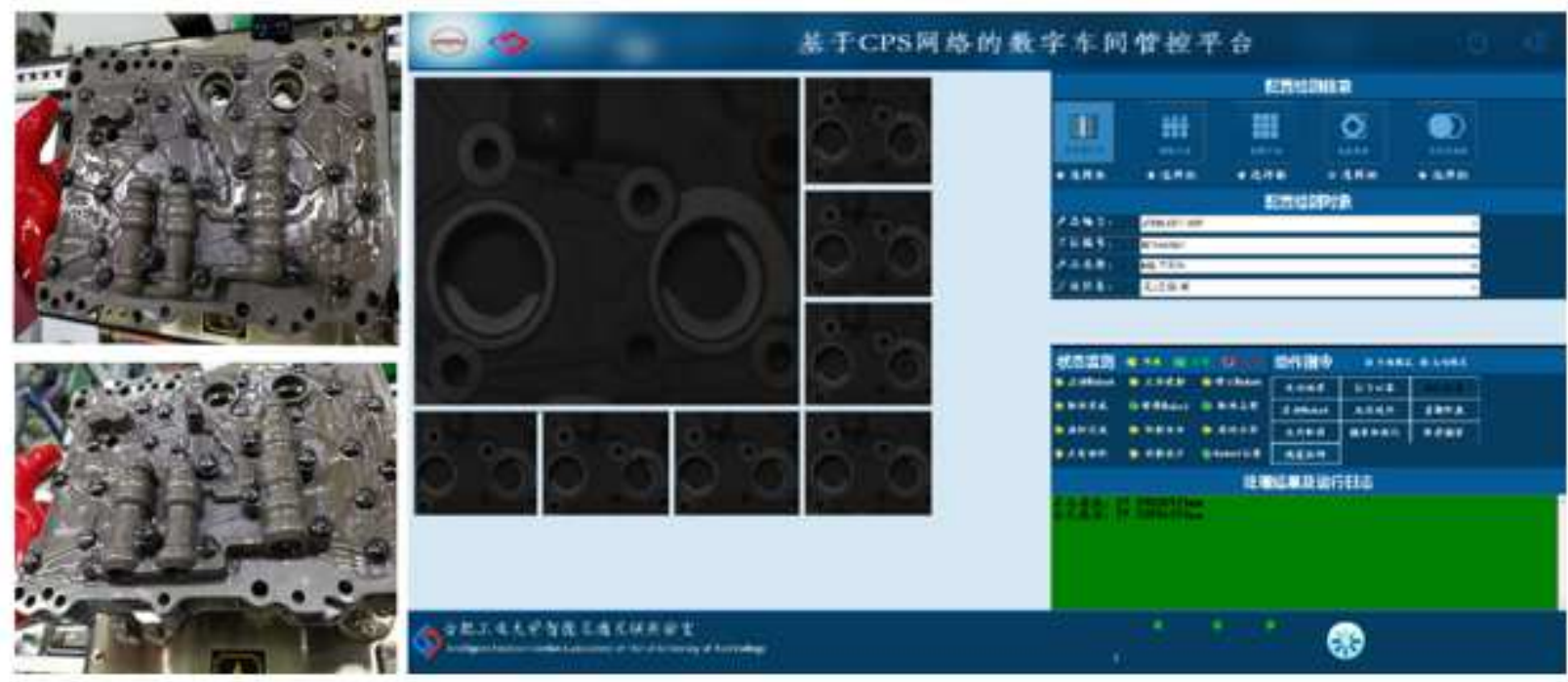


Figure 6

Dynamic scene space and assembly path planning

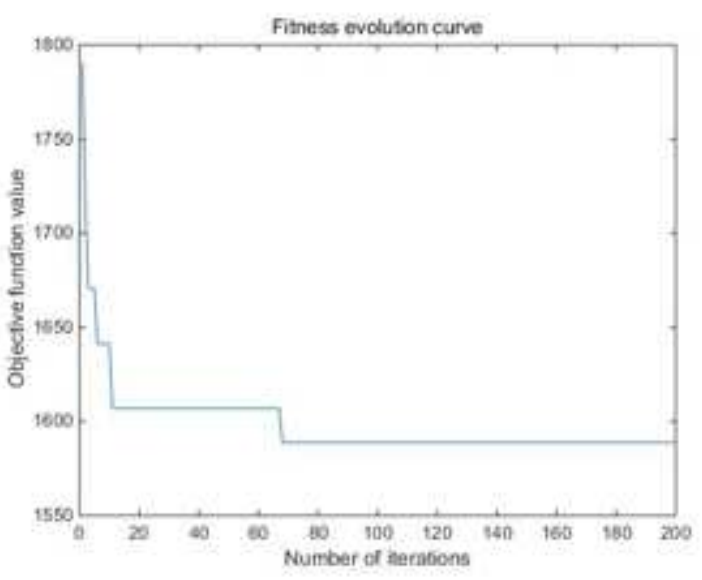

a

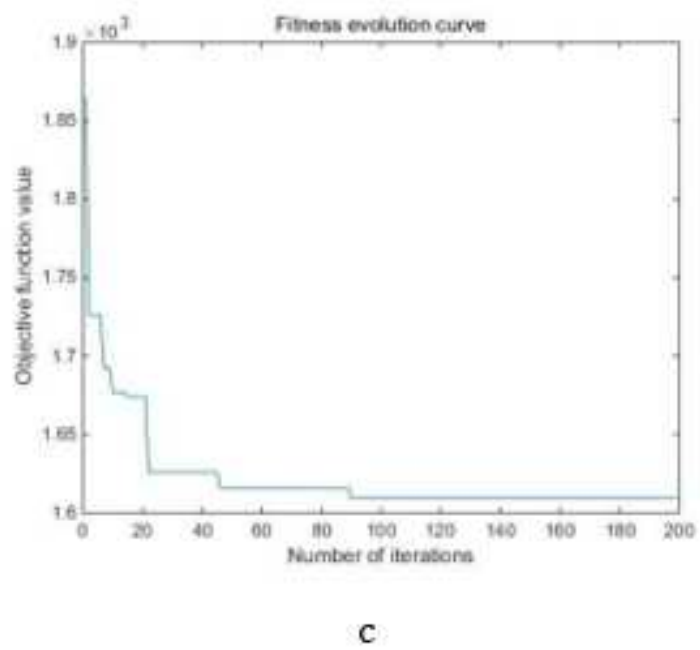

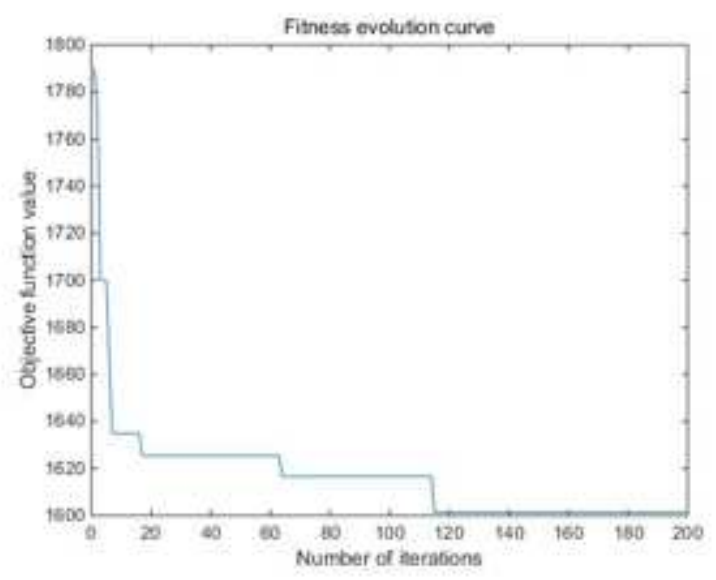

b

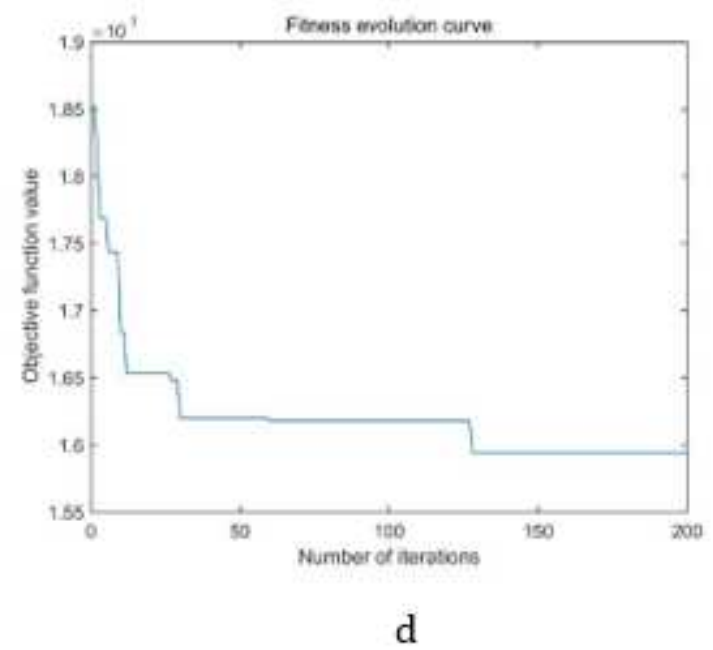

\section{Figure 7}

Curve of shortest path length of algorithm in this paper(a), ACS algorithm(b), control group 2(c) and control group 3(d) 